\title{
An Optical Excursion from Micro-Fibers to Semiconductor Micro-Lasers
}

\author{
Grace Chern, Yong-Le Pan, and Richard K. Chang \\ Dept. of Applied Physics, Yale University, New Haven, Connecticut 06520-8284
}

Our talk will start with a review of the first observation of a series of sharp peaks superimposed on the much broader fluorescence and spontaneous Raman peaks from a uranium oxide fiber and a regular optical fiber, respectively [1]. At first, these numerous and not all spectrally resolved sharp peaks were thought to be noise. Soon afterwards, these peaks were understood to be cavity enhanced spontaneous emission at particular wavelengths. Fibers can be treated as 2-d cavities. As is the case for 3-d cavities where Lorenz/Mie theory is used, all resonance peaks are referred to morphologydependent resonances (MDRs) or whispering gallery modes (WGMs) [2]. The WGMs are envisioned as standing waves, which can be decomposed into clockwise (CW) and counterclockwise (CCW) circulating waves internal to the cylinder's circumference. By symmetry, these two counter-propagating waves are degenerate, except for a phase factor. In the ray model, these $\mathrm{CW}$ and $\mathrm{CCW}$ waves are incident at angles greater than the total-internal-reflection angle $\left(\theta_{\mathrm{c}}=\sin ^{-}\right.$ $\left.{ }^{1}(1 / \mathrm{n})\right)$. Associated with the evanescent wave is a surface wave that is tangent to the interface [3]. Leakage out of the cavity is through the surface-wave component because the curved interface converts the surface wave into a propagating wave, which we detect as the far-field intensity. The order of the WGM radial component determines the penetration length of the evanescent field outside the cylinder. The $n$th order radial component has $n$ peaks (when angle $\phi$ is averaged) as the distance along the radius increases from 0 to $a$. For the $1^{\text {st }}$ order WGM, this mode will have the shortest penetration length, the lowest amount of surface waves, and, hence, the highest Q-value. In the ray picture, the incident angle for the $1^{\text {st }}$ order is largest compared to higher orders for the radial function. WGM peaks in the fluorescence and Raman spectra are referred to as output resonances.

Input resonances occur when the incident wavelength coincides with a WGM. Usually, input resonances are probed by measuring the elastic scattering spectra at a fixed angle. The overall fluorescence and Raman intensity increases when the input resonance is reached as the incident wavelength is finely scanned. Double resonance refers to the special case when the input and output wavelength are both at some different MDR. Elastic scattering spectra have been investigated with the incident beam focused just outside the fiber, which even with the optimally adjusted "impact parameter" for best coupling, is still, on the absolute scale, poorly coupled with the evanescent wave of the WGMs. Only the lower Q-value WGMs have sufficient coupling with the externally focused incident beam. In the elastic scattering spectrum [3], the broad peaks associated with the lower Q $\left(10^{2}\right.$ or lower) are hard to distinguish from the background changes due to other causes. The very sharp peaks associated with $\mathrm{Q}>10^{4}$ are not observable due to poor coupling of the incident beam with these higher Q WGMs. Improved coupling to the higher Q-value can be achieved by converting part of the focused incident wave to an evanescent wave by using a tapered fiber, cladding thinned fiber, slant-cut fiber, or prism [4]. A review of the WGMs observed in the elastic scattering spectra will be given. 
The degeneracy of the CW and CCW waves of the WGMs is lifted if the circular cross-section of the fiber is distorted into a spiral cross-section with its radius changing as $r(\phi)=r_{o}(1+\varepsilon \phi / 2 \pi)$ (see Fig.1). The CCW wave directly encounters the "notch" and a large fraction of this wave can escape via transmission. The $\mathrm{CW}$ wave directly encounters the $90^{\circ}$ inner notch corner and a small fraction of this wave leads to scattering, with a fraction of this being converted to a $\mathrm{CCW}$ wave. Unidirectional laser emission at $404 \mathrm{~nm}$ has been achieved with InGaN multiple quantum-well diodes (see Fig. 2), in the form of a $1.5 \mu \mathrm{m}$ high $\mu$-pillar with a spiral-shaped cross-section, having $\mathrm{r}_{\mathrm{o}} \approx 250$ $\mu \mathrm{m}[5]$. For the $\mu$-pillars with the spiral cross-section, feedback is provided by the CW waves, which are on multi-WGMs with total-internal reflections at the $\mu$-pillar sidewall. The output coupling is dependent on the small amount of conversion from CW to CCW waves at the $90^{\circ}$ inner notch corner and the transmission through the notch. The lowest lasing threshold is achieved when the optically or electrically pumped region has good spatial overlap with the spatial distribution of the CW wave. Thus, close-to-the-edge ring-shaped pumping is the most effective configuration. We will present our latest result on the lasing properties of such a spiral-shaped $\mu$-pillar blue laser. The possibility of stimulated Raman scattering taking place with ring-shaped optical pumping will be speculated.

\section{References}

[1] R.K. Chang et al., J. Raman Spectrosc. 10, 178 (1981).

[2] J.F. Owen et al., Opt. Lett. 6, 540 (1981)

[3] A.W. Poon et al., Opt. Lett. 26, 23 (2001)

[4] Y.L Pan et al., Appl. Phys. Lett. 82, 4 (2003)

[5] G.D. Chern et al., "Uni-directional lasing from InGaN multiple quantum-well spiral-shaped micropillars," submitted to Appl. Phys. Lett. (2003)

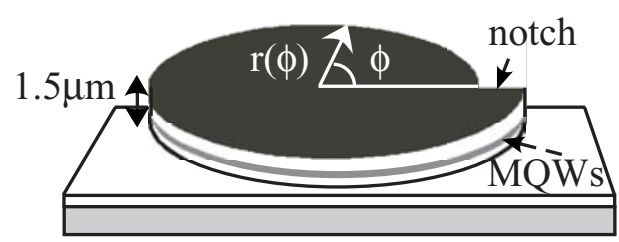

Figure 1. Diagram of micropillar with spiral-shaped cross-section defined by $r(\phi)=r_{o}(1+\varepsilon \phi / 2 \pi)$.

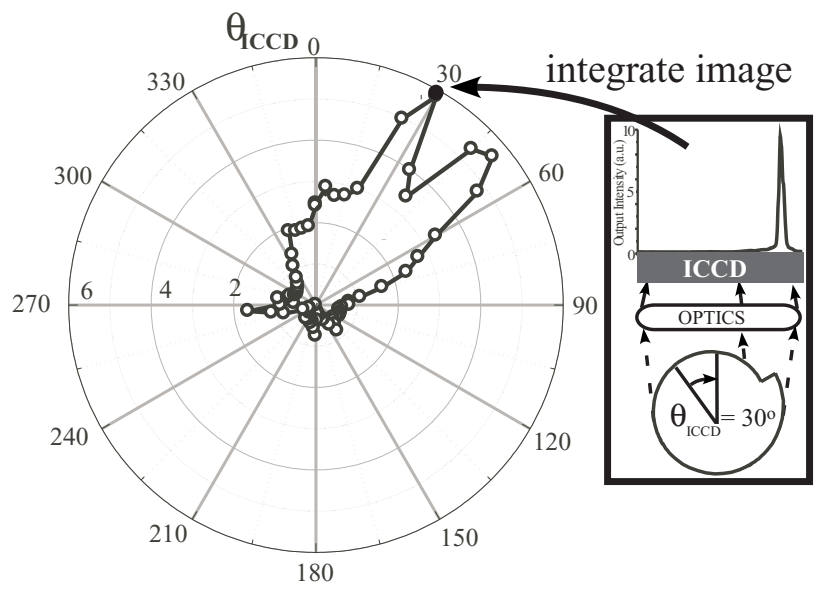

Figure 2. Uni-directional far-field emission pattern of spiral micropillar. 